Canadian

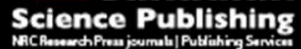

Canadian Journal of Physiology and Pharmacology Revue canadienne de physiologie et pharmacologie

\title{
Adaptation of mitochondrial expression and ATP production in dedifferentiating vascular smooth muscle cells.
}

\begin{tabular}{|r|l|}
\hline Journal: & Canadian Journal of Physiology and Pharmacology \\
\hline Manuscript ID & cjpp-2017-0227.R1 \\
\hline Manuscript Type: & Article \\
\hline Date Submitted by the Author: & 31 -Jul-2017 \\
\hline $\begin{array}{r}\text { Complete List of Authors: } \\
\begin{array}{r}\text { Is the invited manuscript for } \\
\text { consideration in a Special } \\
\text { Issue?: }\end{array}\end{array}$ & $\begin{array}{l}\text { Scheede-Bergdahl, Celena; McGill University, Kinesiology \& Physical } \\
\text { Education; McGill Research Centre for Physical Activity \& Health }\end{array}$ \\
\hline Keyword: & $\begin{array}{l}\text { Atherosclerosis, Mitochondria, Smooth muscle cell dedifferentiation, } \\
\text { OXPHOS, ROS }\end{array}$ \\
\hline &
\end{tabular}

SCHOLARONE $^{\text {m }}$

Manuscripts 


\title{
Adaptation of mitochondrial expression and ATP production in dedifferentiating vascular smooth muscle cells.
}

\author{
Celena Scheede-Bergdahl ${ }^{1,2}$ and Andreas Bergdahl ${ }^{3}$
}

${ }^{1}$ Department of Kinesiology \& Physical Education, McGill University, Montreal, Qc, Canada; ${ }^{2}$ McGill Research Centre for Physical Activity \& Health, McGill University, Montreal, QC, Canada; ${ }^{3}$ Department of Exercise Science, Concordia University, Montreal, Qc, Canada;

Address for Correspondence:

Andreas Bergdahl, $\mathrm{PhD}$

Department of Exercise Science

Concordia University

7141 Sherbrooke Street West

Montreal, Qc, Canada H4B 1R6

Tel: 001-514-848-2424 ext. 5004

E-mail: andreas.bergdahl@ concordia.ca 


\begin{abstract}
Atherosclerosis is one of the leading causes of morbidity and mortality in the Western world. Although the clinical manifestations of this disease are well documented, the etiology and progression remain to be fully understood. Recently, the mitochondria have been implicated in important cellular processes involved in development of atherosclerosis. Despite the link between mitochondria and atherosclerosis, early phase mechanisms of the disease have yet to be elucidated. The aim of this project was to explore the role of mitochondria in vascular smooth muscle (VSMC) dedifferentiation. A murine in-vitro model, involving organ culture of aortic tissue in serum-free media, was used. Mitochondrial function was measured by high resolution respirometry. Proteins associated with the VSMC phenotype switch, as well as mitochondrial density, were assessed by immunoblotting.

The findings show that intrinsic mitochondrial Complex I activity is significantly upregulated during VSMC dedifferentiation. Diminished coupling between phosphorylation and oxidation was also found, indicating a greater ADP:ATP ratio. This data suggests increased leak in the electron transport chain and altered mitochondrial function specifically at Complex I.

This project provides important information regarding the role of mitochondria in the early atherosclerotic process and that detectable changes in mitochondrial function and expression are related to VSMC dedifferentiation.
\end{abstract}

Keywords: Atherosclerosis, smooth muscle cell dedifferentiation, mitochondria, OXPHOS, beta oxidation, ROS, phenotype switch. 


\section{Introduction}

Vascular smooth muscle cells (VSMC) are highly specialized and retain remarkable plasticity, which allows for a transition from a contractile to synthetic phenotype in response to functional demands and external stimuli (Campbell and Campbell, 1985). In their contractile state, VCSM are minimally proliferative and uniquely located in the tunica media of arteries where they regulate blood pressure and flow distribution by means of vessel tonus (Owen GK, 1995). In response to vascular injury, VSMC become dedifferentiated, highly proliferative and migrate to site of the lesion where they mediate important shifts in cell signaling and extracellular matrix production (Hellstrand P, 1998; Cecchettini A et al., 2011). This process of dedifferentiation is a key factor in the atherosclerotic process and involves a morphological change in the cell, as well as an alteration in function and signaling pathways (Campbell and Campbell, 1985; Cecchettini A et al., 2011). Although a number of transcriptional networks that initiate and facilitate the dedifferentiation process have been mapped, the master regulator(s) of VSMC phenotype switch still remain elusive. The clarification of these factors is critical for the prevention and early management of vascular disease.

Although most commonly known for the ability to generate the bulk of ATP, mitochondria also play critical roles in apoptosis, inflammation, metabolism and formation of reactive oxygen species (ROS) generation, all of which feature in atherosclerosis (Yu and Bennett, 2014). Recent work links atherosclerosis to increased mitochondrial-generated ROS, accumulation of mitochondrial DNA (mtDNA) damage and progressive respiratory chain dysfunction (Madamanchi and Runge, 2007; Wang and Tabas, 2014). Furthermore, it has been proposed that mitochondrial dysfunction promotes inflammation and, when coupled with metabolic stresses 
such as cholesterol crystals and fatty acids, leads to a pro-atherosclerotic response that drives plaque development and subsequent disease (Yu and Bennett, 2014). Although there are many evident roles for the mitochondria in the pathogenesis of atherosclerosis, the underpinnings of this relationship are not currently understood (Ong et al. 2013). The interactions between alterations in mitochondrial metabolism and early processes in VSMC dedifferentiation deserve focus as these crucial first steps in the development of atherosclerosis may provide strategies into therapeutic targets (Chiong M et al, 2014).

Reduction-oxidation-dependent regulation, and subsequent production of ROS, play crucial roles in a wide range of biological activities, including atherosclerosis (Wang and Tabas, 2014). It is now known that mitochondrial electron transport chain Complex I is a major site of oxidant production (Murphy, 2009; Brand, 2010), but its contribution to the development of atherosclerosis remain unclear. In order to better comprehend the underpinnings involved in the relationship between the mitochondria and early stage atherosclerosis, the primary aim of this study is to determine whether there are alterations in mitochondrial Complex I function and expression that can, at least partially, explain the early VSMC shifts towards a pro-atherogenic phenotype. Elucidation of these early shifts can provide potential therapeutic targets in a stage of the progression of the disease when reversal is still possible. 


\section{Materials and Methods}

\section{Animal Care}

Male C57Bl/6 mice, 2-3 months old were obtained from Charles River Breeding Farms (St Constant, Quebec) and randomly assigned to either a control (CON) or an experimental (EXP) group. Mice were housed in a thermo-neutral environment $\left(22^{\circ} \mathrm{C}\right)$, on a $12: 12 \mathrm{~h}$ photoperiod, and were provided with free access to water and a standard dry rodent chow. All procedures were approved by the Animal Ethics Committee of Concordia University (Ethics: \#30000259) and were conducted in accordance with guidelines of the Canadian Council on Animal Care.

\section{Tissue Preparation and Culture}

The mice were euthanized by $\mathrm{CO}_{2}$ exposure according to the approved animal protocol after which the aorta was dissected free and kept in nominally $\mathrm{Ca}^{2+}$-free Krebs solution containing (in $\mathrm{mM}$ ): $\mathrm{NaCl} 122, \mathrm{NaHCO}_{3}$ 15.5, $\mathrm{KCl} 4.7, \mathrm{MgCl}_{2}$ 1.2, $\mathrm{KH}_{2} \mathrm{PO}_{4}$ 1.2, glucose 11.5, penicillin 100 $\mathrm{U} / \mathrm{ml}$ and streptomycin $100 \mathrm{Ig} / \mathrm{ml}$. The vessels were used as fresh tissue $(\mathrm{CON})$ or transferred to culture dishes containing culture medium (DMEM/Ham's F12, 1:1, no serum but with antibiotics) and placed in an incubator at $37^{\circ} \mathrm{C}$ under $5 \% \mathrm{CO}_{2}$ in air for $24 \mathrm{~h}(\mathrm{EXP})$. This model is an accepted means of inducing VSMC dedifferentiation, as previously described in the literature (Hellstrand P, 1989; Bergdahl et al. 2005). After culture, the vessels were open up longitudinally and denuded by gentle rubbing of a sponge along the lumen.

\section{Preparation of permeabilized muscle fibers}

Immediately after the aorta was removed from the mouse it was placed in an ice-cold buffer solution (BIOPS) containing (in $\mathrm{mM}$ ): $\mathrm{CaK}_{2}$ EGTA 2.77, $\mathrm{K}_{2}$ EGTA 7.23, $\mathrm{Na}_{2} \mathrm{ATP}$ 5.77, 
$\mathrm{MgCl}_{2} \cdot 6 \mathrm{H}_{2} \mathrm{O}$ 6.56, Taurine 20, Phosphocreatine 15, Imidazole 20, Dithiothreitol 0.5, MES 50, $\mathrm{pH}$ 7.1. After dissection (for CON tissue) or incubation for $24 \mathrm{~h}$ in serum free media (for EXP tissue), the aortic vessels were pulled apart and incubated in $3 \mathrm{ml}$ BIOPS buffer containing 50 $\mu \mathrm{g} / \mathrm{ml}$ saponin for 30 minutes (Larsen et al. 2015). The tissue was then washed in ice-cold buffer (MiR05) for 2 x $10 \mathrm{~min}$. MiR05 contains (in mM): EGTA 0.5, $\mathrm{MgCl}_{2} \cdot 6 \mathrm{H}_{2} \mathrm{O}$ 3.0, K-lactonionate 60, Taurine 20, $\mathrm{KH}_{2} \mathrm{PO}_{4}$ 10, HEPES 20, Sucrose 110, BSA 1g/l, pH 7.1. Measurements of oxygen consumption were performed at $37^{\circ} \mathrm{C}$ using a high resolution respirometer (Oxygraph2k, Oroboros Instruments, Innsbruck, Austria). The respirometric measurements were performed in the buffer MiR05.

\section{Mitochondrial respiratory measurements}

Measurements of oxygen consumption were performed in MiR05 at $37^{\circ} \mathrm{C}$ using a polarographic oxygen sensor (Oxygraph-2k, Oroboros Instruments, Innsbruck, Austria) (Larsen et al. 2015). Approximately 2.0 to $2.5 \mathrm{mg}$ of muscle tissue (wet weight) was placed in either chamber in a cross-sectional design. $\mathrm{O}_{2}$ flux was resolved by DatLab by converting nonlinear changes in the negative time derivative of the oxygen concentration signal. All experiments were carried out in hyperoxygenated levels to avoid $\mathrm{O}_{2}$ diffusion limitations. A previously published (Larsen $\mathrm{S}$ et al. 2012 Pesta D \& Gnaiger E, 2012) sequential substrate addition protocol was used to allow functional dissection of the electron transport system: state 2 respiration (absence of adenylates) was assessed by addition of malate $(2 \mathrm{mM})$ and octanoyl carnitine $(1.5 \mathrm{mM})$, by adding ADP $(5$ $\mathrm{mM}$ ) we could reach state 3 respiration for Complex I. This was followed by addition of glutamate $(10 \mathrm{mM})$ and succinate $(10 \mathrm{mM})$ achieving maximal coupled state 3 respiration with parallel electron input to Complex I and II. Oligomycin $(2 \mu \mathrm{g} / \mathrm{ml})$ was then added to block 
Complex V and thereafter antimycin A $(2.5 \mu \mathrm{M})$ to inhibit Complex III. Finally ascorbate (2 $\mathrm{mM})$ and TMPD $(500 \mu \mathrm{M})$ were added to evaluate Complex IV respiration.

\section{Citrate Synthase Activity}

Citrate synthase activity was determined in whole muscle homogenates using an assay kit (CS0720; Sigma-Aldrich, St. Louis, USA). Total muscle protein was measured by the method of Pierce BCA Assay Kit (Thermo Scientific, Mississauga, ON, Canada), and the protein concentration of all samples equalized. Citrate synthase activity was determined in triplicate based on the formation of TNB (2-nitro-5-thiobenzoic acid) at a wavelength of $412 \mathrm{~nm}$ at $25^{\circ} \mathrm{C}$ on a spectrophotometer (Biochrom WPA Biowave Spectrophotometer, Montreal Biotech, Montreal, Canada). In each well, $15 \mu \mathrm{g}$ of total protein sample was added to a reaction medium containing assay buffer, $30 \mathrm{mM}$ acetyl coenzyme A and $10 \mathrm{mM}$ TNB acid, according to the manufacturer's instructions. The baseline assay solution absorbance was recorded, reactions were initiated by addition of $10 \mu \mathrm{l}$ oxaloacetic acid, and the change in absorbance measured every $10 \mathrm{~s}$ for $2 \mathrm{~min}$.

\section{Protein Extraction, Immunoblotting, and Immunofluorescence}

Procedures were performed as described by Rocha et al. (Rocha et al. 2014). Cell lysates were extracted in lysis buffer containing (in $\mathrm{mM}$ ) $\mathrm{NaCl} 250$, HEPES 50, glycerol 10\%, triton $\mathrm{X}-100$ $1 \%, \mathrm{MgCl}_{2}$ 1.5, EGTA 1, $\mathrm{Na}_{4} \mathrm{P}_{2} \mathrm{O}_{7} 10, \mathrm{NaF} 1, \mathrm{Na}_{3} \mathrm{VO}_{4} 800 \mu \mathrm{M}, \mathrm{pH} 7.5$ and centrifuged at $12,000 \times g$ for $10 \mathrm{~min}$. Supernatant was collected, and protein was measured using Pierce BCA Protein Assay Kit (Thermo Scientific, Mississauga, ON, Canada). $15 \mu \mathrm{g}$ of lysates were separated on a $12.5 \%$ SDS-PAGE and transferred to a nitrocellulose membrane $(0.45 \mu \mathrm{m}, 162$ - 
0115, Bio-Rad) using $10 \mathrm{mM}$ sodium tetraborate buffer. The membranes were blocked in 5\% BSA in TBS-T buffer (10 mM Tris-HCl, pH 7.5, $150 \mathrm{mM} \mathrm{NaCl}, 0.05 \%$ Tween 20) for $1 \mathrm{~h}$ at room temperature followed by overnight incubation at $4{ }^{\circ} \mathrm{C}$ with primary antibodies: Calponin (1:20000, ab46794 Abcam), TRPC1 (1:2000, ACC-010 Alomone labs), Alpha Smooth Muscle Actin (1:2000, ab7817 Abcam) and Voltage Dependent Anion Channel (1:1000, ab14734 Abcam) and Total OXPHOS Rodent antibody cocktail (1:500, MS604 MitoSciences). The blots were washed, incubated with horseradish peroxidase-conjugated secondary antibodies (antimouse, ab6728; Abcam) and visualized with a chemiluminescence system (Immun-Star Chemiluminescent; 1705070; Bio-Rad, Mississauga, ON, Canada). The bands were analyzed using the Image $\mathbf{J}$ software.

\section{Statistics}

Data are presented as means \pm SEM for the immunoblotting and means \pm SE in all other figures. As the data analysis for this study required the comparison between the EXP and CON conditions, a two-tailed Student's t-test was utilized, where $P<0.05$ was considered significant. 


\section{Results}

In this study, we compared tissue that was undergoing VSMC dedifferentiation (EXP), as stimulated by incubation in serum free media, to control tissue (CON).

Tissue dedifferentiation: $24 \mathrm{~h}$ in SFM induced a relative downregulation of the expression of the dedifferentiation marker calponin (EXP $58.6 \pm 14.6 \%$ versus CON tissue at $100 \pm 12.2 \%$, p < 0.05) as depicted in Figure 1. To further indicate tissue dedifferentiation, the amount of another marker, TRPC1, increased in the EXP tissue when compared to CON (EXP: $195.9 \pm 39.4 \%$ percent versus $\mathrm{CON}: 100 \pm 19.7 \%$ versus $\mathrm{p}<0.05$, as shown in Figure 1$)$. The late smooth muscle specific alpha actin differentiation marker demonstrated a downwards trend in the EXP tissue (Figure 1) further confirming that our model induces a shift in VSMC phenotype.

Mitochondrial density: To control for relative differences in mitochondrial content, immunoblotting with an antibody specific for the voltage dependent anion channel (VDAC) was used (Figure 2a). This indicated a significant downregulation in EXP from the CON condition (EXP: $50.2 \pm 14.4 \%$ versus CON: $100 \pm 15.9 \%, \mathrm{p}<0.05)$. This was confirmed through the analysis of citrate synthase activity as shown in Figure $2 b$, which is another commonly used technique for measuring mitochondrial density. Using the kinetics of this enzyme, there was a significant reduction of mitochondrial density in the EXP vessels, which paralleled the data obtained for the VDAC analysis (EXP: $1.21 \pm 0.27 \mathrm{nmol} / \mathrm{mg}$ protein/min versus CON: $4.06 \pm$ $0.42 \mathrm{nmol} / \mathrm{mg}$ protein/min, $\mathrm{p}<0.01)$. 
Mitochondrial proteins: Normalized to total amount of protein, the expression of the mitochondrial subunits were all downregulated in the EXP vessels (Figure 3a). Complex I showed a significant downregulation in the EXP model (EXP: $27 \pm 6.4 \%$ versus CON: $100 \pm$ $8.6 \%, \mathrm{p}<0.001)$ while Complex II in the cultured condition indicated a non-significant trend towards downregulation (EXP: $54.6 \pm 15.4 \%$ versus CON: $100 \pm 18.4 \%, \mathrm{p}=0.13$ ). Complex III was also significantly lower in the EXP condition (EXP: $38.5 \pm 9.0 \%$ versus CON: $100 \pm 11.7 \%$, $\mathrm{p}<0.001)$ which is in line with the data for Complex V (EXP: $43.0 \pm 4.8 \%$ versus CON: $100 \pm$ $13.3 \%, \mathrm{p}<0.01)$. The expression of Complex IV indicated a downwards trend in the EXP vessels (EXP: $58.2 \pm 15.7 \%$ versus $\mathrm{CON}$ : $100 \pm 11.7 \%, \mathrm{p}=0.06$ ). When normalized to $\mathrm{CS}$ activity (Figure 3b) no statistical differences were detected although there was a trend towards an increased expression of Complex II and Complex IV in the EXP model. The amount of Complex I (EXP: $91 \pm 21.5$ versus CON: $100 \pm 8.6)$, Complex III (EXP: $126 \pm 34.7$ versus CON: $100 \pm$ 12.9) and Complex V (EXP: $144 \pm 16.2$ versus CON: $100 \pm 13.3)$ were unaffected by the culture in SFM when the values are normalized to CS activity. Complex II (EXP: $183 \pm 51.5$ versus CON: $100 \pm 18.4$ ) and Complex IV (EXP: $195 \pm 52.5$ versus CON: $100 \pm 13.0)$ indicated a strong, yet nonsignificant trend towards upregulation when normalized to CS activity.

Mitochondrial respiration: To investigate respiratory capacities following VSMC phenotype switch, we studied the oxygen consumption rates, normalized to CS activity. As seen in Figure 4b, the dedifferentiated VSMC demonstrated a significant upregulation (EXP: $1.51 \pm 0.19$ $\mathrm{pmol} / \mathrm{s} / \mathrm{mg} / \mathrm{CS}$ activity versus CON: $0.44 \pm 0.06 \mathrm{pmol} / \mathrm{s} / \mathrm{mg} / \mathrm{CS}$ activity, $\mathrm{p}<0.01)$ in OXPHOS capacity with the Complex I linked substrate malate (basal, ADP-restricted). The acceptor control ratio (ACR) (Figure 4c), representing the degree of coupling between oxidation and 
phosphorylation, was significantly downregulated in the EXP tissue (EXP: $2.45 \pm 0.29$ $\mathrm{pmol} / \mathrm{s} / \mathrm{mg} / \mathrm{CS}$ activity versus CON: $4.64 \pm 0.88 \mathrm{pmol} / \mathrm{s} / \mathrm{mg} / \mathrm{CS}$ activity, $\mathrm{p}<0.05)$. The lipid OXPHOS capacity in Figure 5a was significantly higher in the EXP compared with CON (EXP: $3.55 \pm 0.29 \mathrm{pmol} / \mathrm{s} / \mathrm{mg} / \mathrm{CS}$ activity versus CON: $1.98 \pm 0.49 \mathrm{pmol} / \mathrm{s} / \mathrm{mg} / \mathrm{CS}$ activity, $\mathrm{p}<0.05)$. The EXP tissue showed a significantly higher OXPHOS capacity with Complex I linked substrates when compared to the CON condition (EXP: $4.25 \pm 0.46 \mathrm{~mol} / \mathrm{s} / \mathrm{mg} / \mathrm{CS}$ activity versus CON: $2.41 \pm 0.59 \mathrm{pmol} / \mathrm{s} / \mathrm{mg} / \mathrm{CS}$ activity, $\mathrm{p}<0.05$ ) (Figure 5b). Similarly, a more detectable difference between EXP and CON was observed in OXPHOS capacity, as determined by Complex I and II linked substrates (EXP: $9.00 \pm 0.47 \mathrm{pmol} / \mathrm{s} / \mathrm{mg} / \mathrm{CS}$ activity versus CON: $5.08 \pm$ $0.68 \mathrm{pmol} / \mathrm{s} / \mathrm{mg} / \mathrm{CS}$ activity, $\mathrm{p}<0.01)$. This data is depicted in Figure 5c. No differences were found in Complex IV OXPHOS capacity between EXP and CON (data not shown). There was a non-significant trend toward an upregulation of the residual oxygen consumption (ROX) in the EXP tissue (data not shown). Mitochondrial leak, as depicted in Figure 6a, is estimated by antimycin A flux rates subtracted from oligomycin flux rates and indicates a significant increase in the EXP VSMC (EXP: $5.06 \pm 0.22 \mathrm{pmol} / \mathrm{s} / \mathrm{mg} / \mathrm{CS}$ activity versus CON: $3.31 \pm 0.58$ $\mathrm{pmol} / \mathrm{s} / \mathrm{mg} / \mathrm{CS}$ activity, $\mathrm{p}<0.05)$. No differences were seen in substrate control ratio for succinate $\left(\mathrm{GM}_{3} / \mathrm{GMS}_{3}\right)$ or the lipid coupling control ratio (Figure 6b). Furthermore, there was no difference (Figure 6c) in respiratory control ratio (RCR) between the CON and EXP tissue, indicating no change in capacity for substrate oxidation and ATP turnover. 


\section{Discussion}

The major finding of this study is that, despite a lower mitochondrial density, basal respiration in Complex I is enhanced in the dedifferentiated tissue (EXP), when compared to control. We also found, in the dedifferentiated tissue, a diminished coupling between phosphorylation and oxidation, as well as an increased ability for lipid oxidation, indicating a shift in metabolism from glucose based substrates to free fatty acids. Despite these alterations in mitochondrial content and function, there was no difference in substrate handling between the two conditions. This indicates that the higher respiration is not caused by an increased use of substrates but rather from an enhanced ability for the mitochondria to extract energy from fuel sources. This "overdrive" of Complex I that occurs during VSMC phenotype shift, as well as the greater ADP:ATP ratio, may be the sources of the elevated ROS previously reported in cardiovascular disease (Panth $\mathrm{N}$ et al., 2016), as well as the early pro-atherogenic shifts that lead to disease progression (Förstermann U et al., 2017).

Although a great deal of advancement has been made in the understanding of the atherosclerotic process, the precise underpinnings of this disease remain poorly elucidated. Part of the issue lies in the experimental models traditionally used; cell culture or injury models elicit rapid changes in the atherosclerotic process that do not support investigation of subtle shifts that occur in the disease progression (Buck 1977; Todd and Friedman 1978). In light of these methodological issues, this study uses organ culture in serum-free media, in order to promote a slower progression of vascular injury and subsequent VSMC dedifferentiation (Koo and Gotlieb, 1991). This allows for an opportunity to better study the mechanisms that lie at the root of early atherosclerotic development (Soyombo et al. 1990; Bergdahl et al. 2005). 
Our data shows that that the basal metabolic rate, normalized to CS activity, was significantly upregulated in the EXP tissue. Basal metabolic rate is affected by both the degree of transmembrane proton leak and the total number of mitochondria (Brand et al. 2005), which was shown in our study to be reduced. The higher Complex I stimulated/ADP restricted respiration in the EXP tissue is most likely due to an increase in transmembrane proton leak. This hypothesis is strengthen by the elevated leak observed in the EXP tissue, as depicted in Figure 6a. Interestingly, the increase in EXP oxidative capacity detected in this study occurs despite a significant decrease in the coupling between oxidation and phosphorylation, as suggested by the ACR values. This indicates that the EXP tissue has a lower oxidation:phosphorylation ratio, which could be compensated through elevated ADP stimulated respiration. This is the exact phenomenon that we report in Figures $5 \mathrm{~b}$ and $5 \mathrm{c}$ for Complex I and Complex II, respectively. The overall respiration is further augmented in the dedifferentiated tissue due to an increase in lipid oxidation as tested by the medium long fatty acid octanoylcarnitine. Addition of free fatty acids contribute additional reducing equivalents, not originating from malate, which are subsequently fed into the electron transport chain. A similar response did not occur in the CON tissue, suggesting that the increased ATP production is most likely localized in the malate, glutamate and succinate dehydrogenases and not on the level of the electron transport chain and the phosphorylation system. This indicates a higher overall intrinsic mitochondrial oxidative capacity which is exactly what we see when we normalize to the citrate synthase activity (Figure $2 b)$. 
Recent work has attempted to identify the origins of the atherosclerotic process. Mitochondrial dysfunction, accumulation of mitochondrial DNA damage and progressive respiratory chain impairments have been pinpointed as some of the earliest contributing factors to the development of atherosclerosis (Madamanchi and Runge, 2007). Despite these observations, it is not known whether these factors cause or result from excess mitochondrial ROS generated during the progression of atherosclerosis (Wang and Tabas, 2014). The data presented here show that accelerated Complex I respiration occurs early in VSMC dedifferentiation (Figures 4b and 5b) and can thus explain the source of the ROS associated with the detrimental effects of atherogenesis on the mitochondria. This is further supported by the decreased coupling between phosphorylation and oxidation that we observed in the dedifferentiated tissue (Figure 4c), indicating a potential leak or leaks in the electron transport chain. This observation is supported by previous work that shows reduced ATP production through oxidative phosphorylation in a pro-atherogenic diabetic model (Ahn SY et al., 2010). In a previous study, morphofunctional alterations in VSMC, linked to atherogenesis, were resolved by a reduction of inflammation and oxidative stress via antioxidant administration (Baez M del C et al., 2014).

We also report that mitochondrial density is lower in the dedifferentiated tissue when compared to control although, under basal conditions, respiration is highly upregulated. It is not clear precisely why this diminishment of mitochondrial density occurs, although past work has indicated autophagy and increased mitochondrial fission during the VSMC shift from contractile to synthetic phenotype (Salabei and Hill, 2013). In another study, oxidized LDL, a known inducer of atherosclerosis, was shown to decrease the expression of mitochondrial Complex subunits (Ahn SY et al., 2010), which is similar to the data presented here. Increased basal 
respiration may indicate the need for the remaining mitochondria to compensate for the reduced mitochondrial content that results from the VSMC shift, thus increasing metabolism. This may be due to- and regulated by- altered $\mathrm{Ca}^{2+}$ handling, which is a process linked with mitochondrial changes associated with VSMC phenotype shifts (Chiong M et al., 2014). It has previously been shown that, besides preserving the morphology of the vessel, serum stripped organ culture that is in presence of an intact extracellular matrix, induces similar reductions in contractile proteins and $\mathrm{Ca}^{2+}$ handling seen in early stages of VSMC dedifferentiation (Dreja et al. 2001; Kumar et al. 2006). These latter changes may, in fact, precede altered contractile properties and could play a role in the mitochondrial modulation process itself. We demonstrate an increase in TRPC1 expression (Figure 1) which has previously been shown in VSMC undergoing dedifferentiation (Bergdahl et al. 2005).

Our data also demonstrates that, as VSMC progress towards a more synthetic phenotype, an increased reliance on fatty acids as a metabolic source. This has also been previously hypothesized, where this increase in fatty acid oxidation is thought to be required for rapid division, migration of the synthetic cell, as well as the energy requirements needed for the synthesis and secretion of extracellular matrix components (Salabei JK and Hill BG, 2013).

In conclusion, the present study supports the hypothesis that intrinsic mitochondrial dysfunction is implicated in the early VSMC phenotype switch that is crucial for neointima formation. While the related reduction in contractile filaments has been studied in detail, relatively little is known about how the mitochondria affect this process. We report that the mitochondrial function in dedifferentiated VSMC, when corrected for organelle density, is associated with higher ADPrestricted as well as ADP-driven respiration. Our data also suggests that mitochondrial function 
and bioenergetics are important factors in VSMC dedifferentiation. We propose that the reported mitochondrial modifications precede or coincide with structural VSMC changes and that this could be of pathogenic importance in the development of vascular disease. Elucidation of these early phase VSMC shifts associated with atherosclerosis will allow for strategic therapeutic or pharmacological interventions, prior to the full manifestation of the disease. Future work with ETC Complex blockades will allow for a clearer understanding of whether the phenotype shift precedes or is due to alterations in mitochondrial function.

\section{Acknowledgements}

We are very grateful to the Concordia University Animal Care Facility Manager Aileen Murray and her staff for the housekeeping and caregiving they provided throughout this project. Concordia University provided the funds for this project.

\section{References}

Ahn SY, Choi YS, Koo HJ et al. 2010 Mitochondrial dysfunction enhances the migration of vascular smooth muscle cells via suppression of Akt phosphorylation. Biochimica et Biophysica Acta 1800: 275-281.

Baez M, Taran M, Llorens C et al. 2014 Mitochondrial Morphofunctional Alterations in Smooth Muscle Cells of Aorta in Rats. ISRN Cardiology. Vol 2014 Article ID 739526, 6 pages.

Bergdahl A, Gomez MF, Wihlborg AK et al. 2005 Plasticity of TRPC expression in arterial smooth muscle: correlation with store-operated $\mathrm{Ca}^{2+}$ entry. Am J Physiol Cell Physiol. 288(4):C872-80 
Brand MD, Pakay JL, Ocloo A et al. 2005. The basal proton conductance of mitochondria depends on adenine nucleotide translocase content. Biochem J. 392(Pt 2):353-62.

Brand, M. D. 2010 The sites and topology of mitochondrial superoxide production. Exp. Gerontol. 45, 466-472

Buck RC. 1977. Organ culture of rat aorta: a scanning and transmission electron microscopic study. Exp Mol Pathol. 26(2):260-76

Campbell GR and Campbell JH 1985. Smooth muscle phenotypic changes in arterial wall homeostasis: implications for the pathogenesis of atherosclerosis. Exp and Mol Pathology. 42: 139-162.

Cecchettini A, Rocchiccioli S, Boccardi C et al. 2011 Vascular smooth muscle cell activation: proteomics point of view. International Review of Cell and Molecular Biology, Volume 288. Chapter 2:43-99.

Chiong M, Cartes-Saavedra B, Norambuena-Soto I et al. 2014. Mitochondrial metabolism and the control of vascular smooth muscle cell proliferation. Frontiers in Cell and Developmental Biology, Vol 2, article 72

Dreja K, Bergdahl A, Hellstrand P. 2001. Increased store-operated Ca2+ entry into contractile vascular smooth muscle following organ culture. J Vasc Res. 38(4):324-31

Förstermann U, Xia N and Li H 2017. Roles of Vascular Oxidative Stress and Nitric Oxide in the Pathogenesis of Atherosclerosis. Circ Res. 120(4): 713-735 
Hellstrand P. 1998. Long-term effects of intracellular calcium and growth factors on excitation and contraction in smooth muscle. Acta Physiol Scand 164: 637-644.

Koo EW and Gotlieb AI. 1991 Neointimal formation in the porcine aortic organ culture. I. Cellular dynamics over 1 month. Lab Invest 64:743-753.

Kumar B, Dreja K, Shah SS et al. 2006. Upregulated TRPC1 channel in vascular injury in vivo and its role in human neointimal hyperplasia. Circ Res. 98(4):557-63.

Larsen S, Wright-Paradis C, Gnaiger E et al. 2012. Cryopreservation of human skeletal muscle impairs mitochondrial function. CryoLetters 33(3), 169-175

Larsen S, Scheede-Bergdahl C, Whitesell T et al. 2015. Increased intrinsic mitochondrial respiratory capacity in skeletal muscle from rats with streptozotocin-induced hyperglycemia. Physiol Rep. 2015 3(7)

Madamanchi NR and Runge MS. 2007. Mitochondrial dysfunction in atherosclerosis. Circ Res. 100:460-473

Murphy, M P. 2009. How mitochondria produce reactive oxygen species. Biochem. J.417, 113.

Ong SB, Hall AR and Hausenloy DJ. 2013. Mitochondrial dynamics in cardiovascular health and disease. Antioxidants and Redox Signaling Vol 19. 4:400-414.

Owens GK. 1995. Regulation of differentiation of vascular smooth muscle cells. Physiol Rev 75: 487-517. 
Panth N, Paudel KR and Parajuli K. 2016. Reactive Oxygen Species: A Key Hallmark of Cardiovascular Disease. Adv. Med 2016: 9152732

Pesta D and Gnaiger E. 2012. Chapter 3 Mitochondrial Bioenergetics: Methods and Protocols, Methods in Molecular Biology, Vol 810. 25-58

Rocha C, Scheede-Bergdahl C, Whitesell T et al. 2014. Implications of Apolipoprotein E Deficiency on Cardiac Mitochondrial Oxygen Consumption in a Young Mouse Model. European Journal of Cardiovascular Medicine. Vol 3(1): 394-400

Salabei JK and Hill BG. 2013. Mitochondrial fission induced by platelet-derived growth factor regulates vascular smooth muscle cell bioenergetics and cell proliferation. Redox Biology 1:542-551.

Soyombo AA, Angelini GD, Bryan AJ et al. 1990. Intimal proliferation in an organ culture of human saphenous vein. Am J Pathol 137:1401-1410.

Todd ME and Friedman SM. 1978. The rat-tail artery maintained in culture: an experimental model. In Vitro 14(9):757-70

Yu EPK and Bennett MR. 2014. Mitochondrial DNA damage and atherosclerosis. Trends in Endocrinology and Metabolism, Vol 25, No 9:481-487.

Wang Y and Tabas I. 2014. Emerging roles of mitochondria in atherosclerotic lesions: causation or association? J Atheroscler Thromb 21(5):381-390. 


\section{Figure legends}

Figure 1: Detection of known protein contractile markers indicate that the expression of calponin is downregulated while the expression of TRPC1 is upregulated in the dedifferentiated smooth muscle cells. The expression of the late marker SM alpha actin only indicates a trend towards downregulation. This immunoblot data demonstrate that the smooth muscle cells from the serum free organ culture have undergone early phenotype shift. Bottom of figure: representative blots and loading control for all proteins. Data are means \pm SEM. $*=\mathrm{P}<0.05$

Figure 2: Determination of mitochondrial density using the voltage dependent anion channel expression (2a) as well as citrate synthase activity (2b) demonstrated a significant downregulation of the amount of mitochondria in the tissue that had been cultured in serum free media for $24 \mathrm{~h}$. Representative blot and loading control. Data are means \pm SEM. $*=\mathrm{p}<0.05$ and $* * *=\mathrm{P}<0.001$

Figure 3: Immunoblotting of Complex I to Complex V using the total OXPHOS rodent antibody cocktail from MitoSciences (MS604). In 3a, when normalized to total amount of protein, all Complexes demonstrated a downregulation following phenotype shift with significance in Complex I, III and V. 3b indicates no significant change for all Complexes when normalizing the expression to CS activity. Representative blots in $3 \mathrm{c}$. Data are means $\pm \mathrm{SE}$. $* *=\mathrm{P}<0.01$ 
Figure 4: 4a demonstrates representative Oxygraph respiration of CON tissue. The basal, ADP restricted respiration induced by the Complex I stimulating Malate indicate an upregulation in the synthetic smooth muscle cells (4b). There was a decrease in the acceptor control ratio (ACR) when comparing to the control tissue (4c) signifying a decrease in the coupling between the oxidation and phosphorylation in the dedifferentiated tissue. Data are means \pm SE. $*=p<0.05$ and $* *=\mathrm{P}<0.01$.

Figure 5: Intrinsic respiratory capacity (mitochondrial respiratory capacity per mg tissue divided by CS activity). 5a lipid OXPHOS capacity; 5b OXPHOS capacity with Complex I linked substrates; 5c OXPHOS capacity with Complex I and II substrates. Data are means \pm SE. * $=$ $\mathrm{p}<0.05$ and $* *=\mathrm{P}<0.01$

Figure 6: The mitochondrial LEAK estimated as antimycin A flux rates subtracted from oligomycin flux rates indicates a significant upregulation in the synthetic smooth muscle cells (6a). Substrate control ratio $\left(\mathrm{CI}+\mathrm{II}_{\mathrm{P}} / \mathrm{CI}_{\mathrm{P}}\right)(6 \mathrm{~b})$. The respiratory control ratio (RCR) was unaffected following serum free organ culture (6c). Data are means $\pm \mathrm{SE}$. $*=\mathrm{P}<0.05$ 


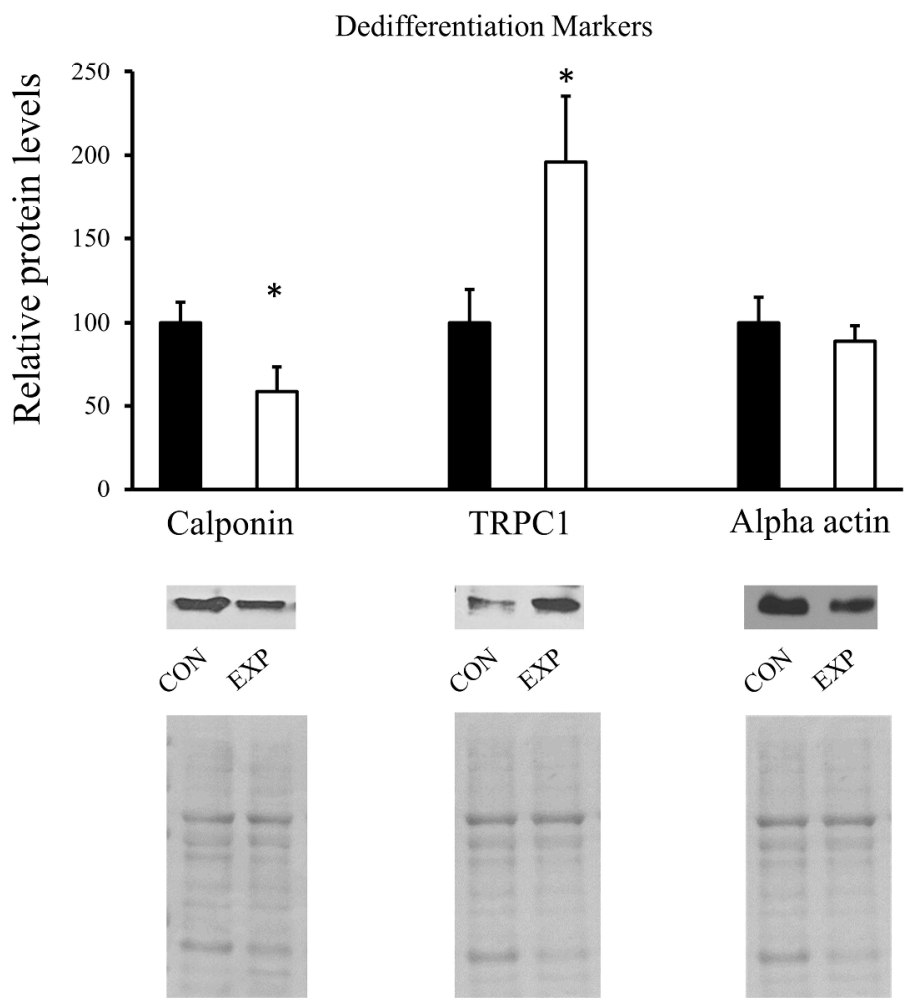

Figure 1

$254 \times 338 \mathrm{~mm}(300 \times 300 \mathrm{DPI})$ 
$2 \mathbf{a}$
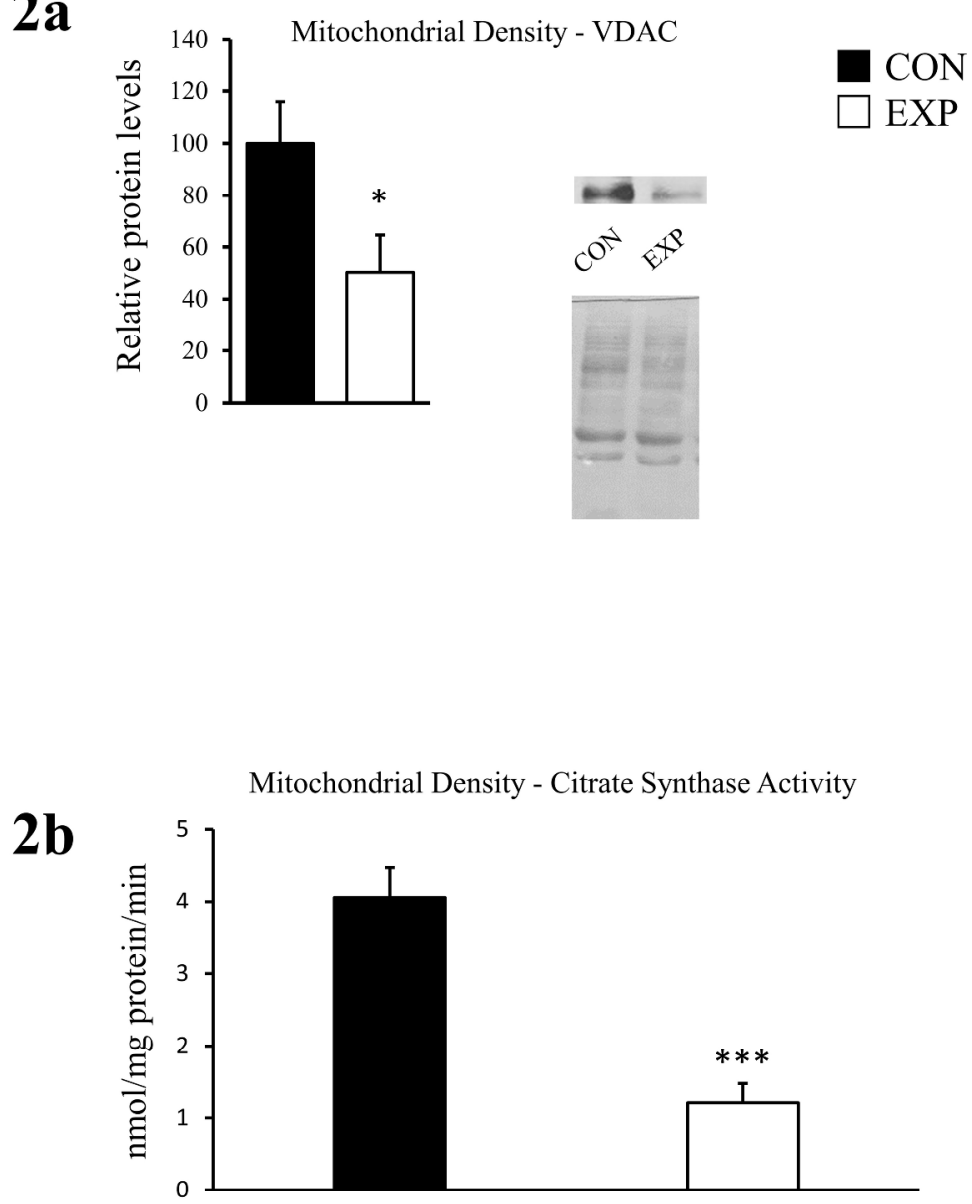

Figure 2

$254 \times 338 \mathrm{~mm}(300 \times 300$ DPI $)$ 
3a

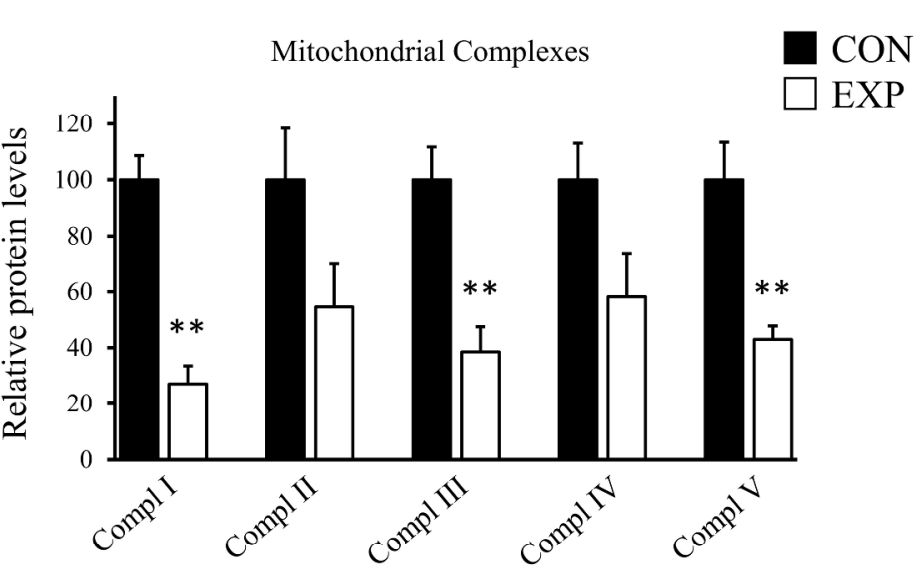

3b

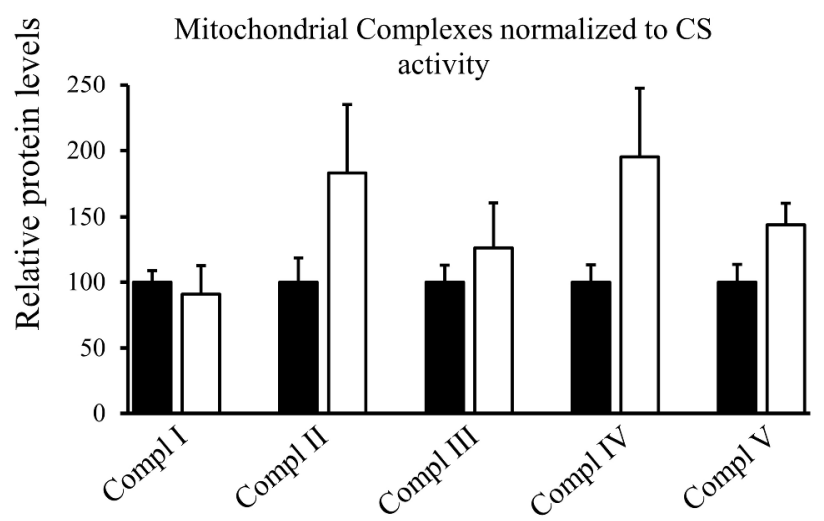

$3 \mathbf{c}$

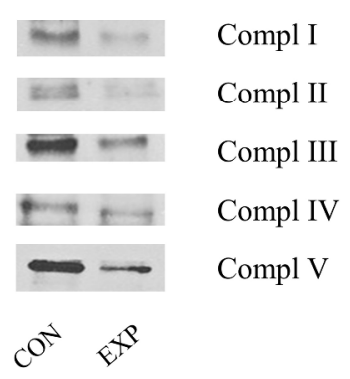

Figure 3

$254 \times 338 \mathrm{~mm}(300 \times 300 \mathrm{DPI})$ 


\section{$4 a$}

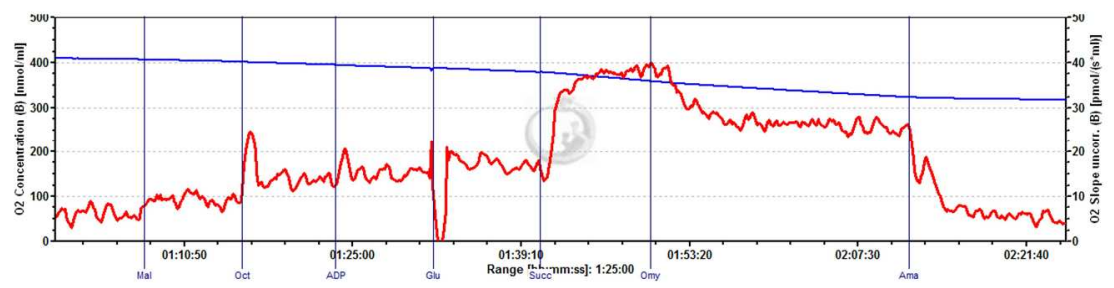

4b

Basal, ADP-restricted respiration

$\square \mathrm{CON}$

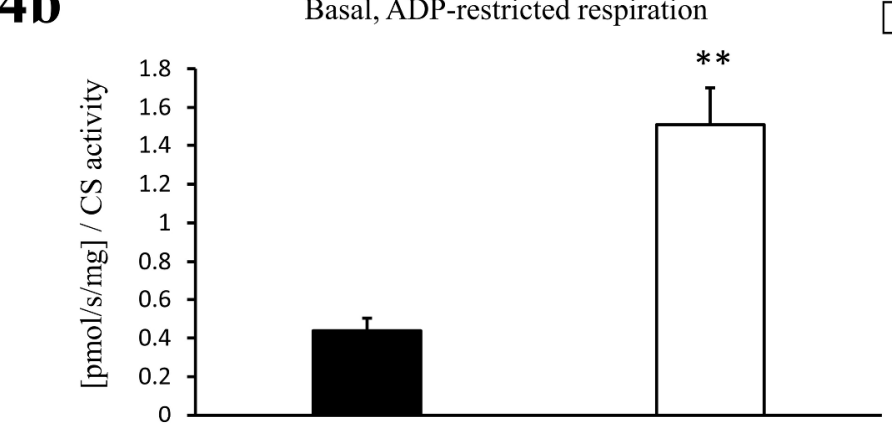

4c Acceptor Control Ratio

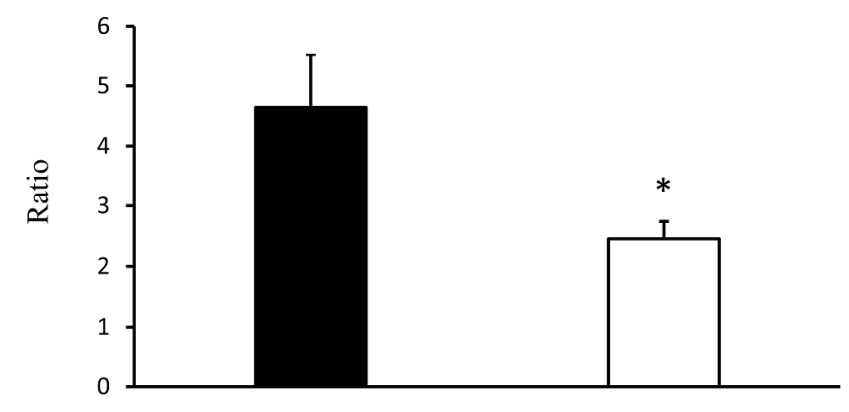

Figure 4 $254 \times 338 \mathrm{~mm}(300 \times 300 \mathrm{DPI})$ 
$5 a$ Lipid OXPHOS Capacity, ETF

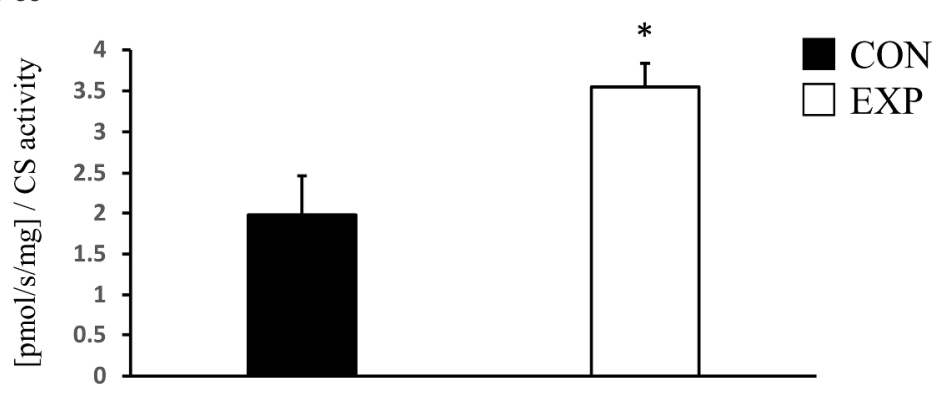

$5 b$

OXPHOS Capacity, $\mathrm{CI}_{\mathrm{P}}$

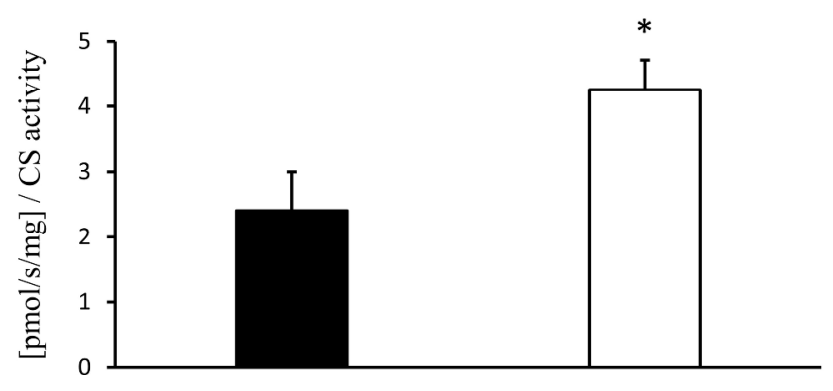

5c

OXPHOS Capacity, $(\mathrm{CI}+\mathrm{CII})_{\mathrm{P}}$

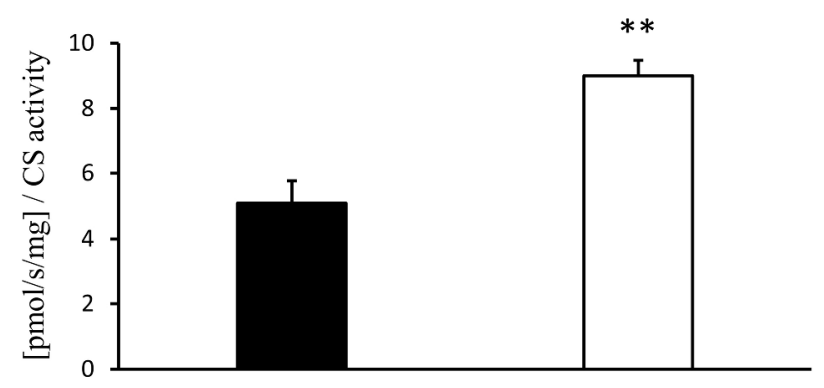

Figure 5

$254 \times 338 \mathrm{~mm}(300 \times 300$ DPI $)$ 

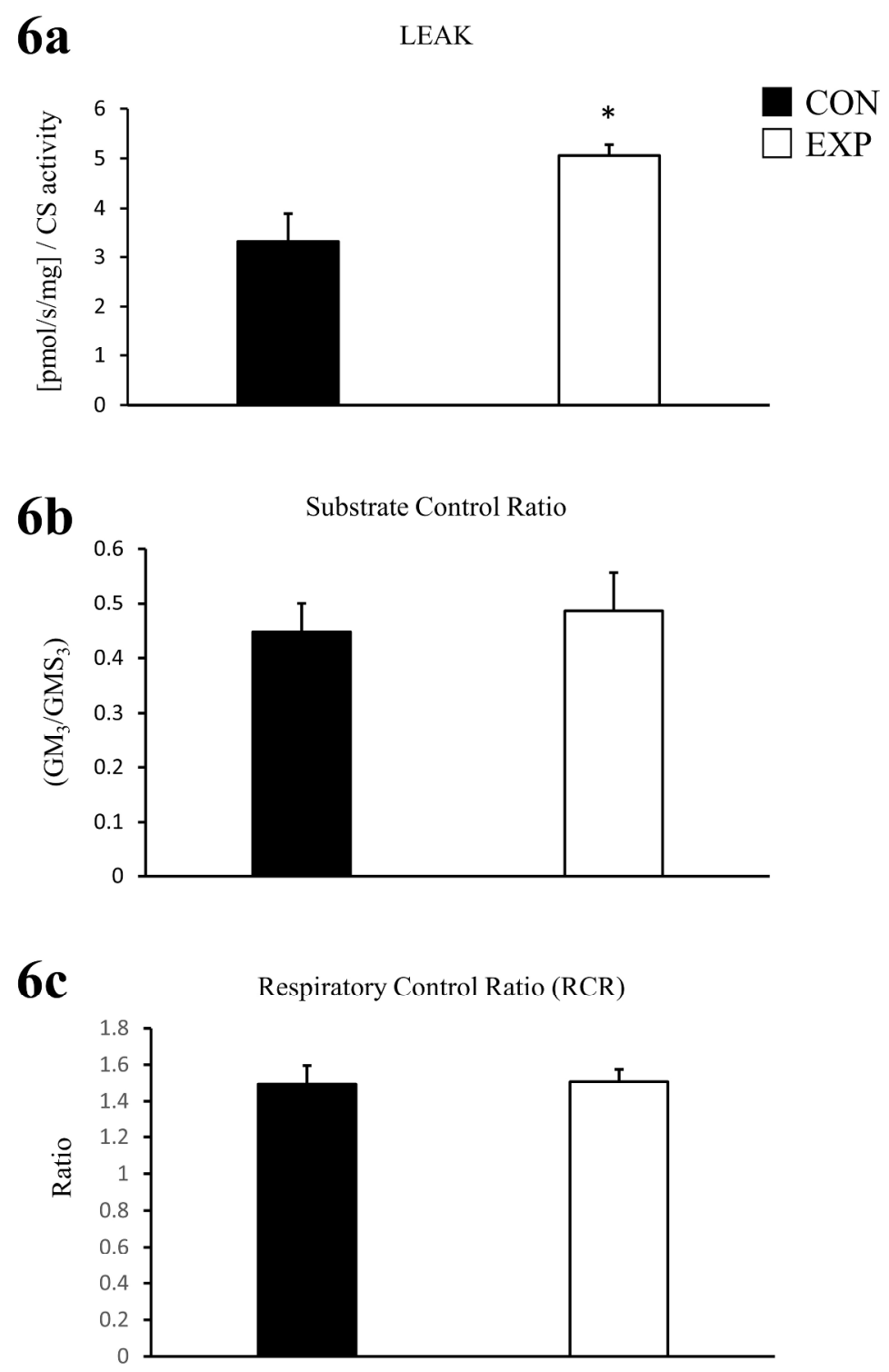

Figure 6 $254 \times 338 \mathrm{~mm}(300 \times 300$ DPI $)$ 